\title{
Políticas de inclusión educativa: el desafío de las universidades públicas hondureñas*
}

\author{
DOI: https://doi.org/10.18046/recs.i34.4227 \\ Policies of Educational Inclusion: The Challenge \\ for Honduran Public Universities \\ Eddy Paz-Maldonado** \\ Universidad Nacional Autónoma de Honduras (Tegucigalpa, Honduras) \\ Ilich Silva-Peña ${ }^{* * *}$ \\ Universidad de Los Lagos (Osorno, Chile)
}

\begin{abstract}
* El artículo es fruto de la línea de investigación Inclusión Educativa en Honduras, desarrollada de manera independiente por los autores. Este trabajo no ha recibido ningún tipo de financiación. Artículo de investigación recibido el 15.08.2020 y aceptado el 02.04.2021.

** Profesor en el Departamento de Pedagogía y Ciencias de la Educación, Universidad Nacional Autónoma de Honduras (UNAH) (Honduras). Magíster en Educación Especial y Psicopedagogía por la Universidad Católica del Maule (Chile). Líneas de investigación vinculadas a la inclusión educativa, atención a la diversidad en la enseñanza superior, colectivos en condición de vulnerabilidad, justicia social y formación docente para la atención a la diversidad. Correo electrónico: eddy.paz@unah.edu.hn ORCID: https://orcid.org/oooo-ooo2-2324-8813

*** Investigador en el Instituto Interuniversitario de Investigación Educativa (Iesed) (Chile) y el Departamento de Educación en la Universidad de Los Lagos (Chile). Profesor de educación general básica y Doctor en Ciencias de la Educación. Director de proyectos nacionales e internacionales. Intereses de investigación: formación docente, justicia social y metodologías de investigación. Correo electrónico: ilichsp@gmail.com ORCID: https://orcid.org/ooooooo1-9118-3989
\end{abstract}




\section{Cómo citar/How to cite}

Paz-Maldonado, Eddy; Silva-Peña, Ilich (2021). Políticas de inclusión educativa: el desafío de las universidades públicas hondureñas. Revista CS, 34, 71-9o. https://doi.org/10.18046/recs.i34.4227 


\section{Resumen}

Hoy asiste un estudiantado diverso a los centros educativos. Como parte de esa diversidad están quienes pertenecen a colectivos vulnerados de manera sistemática. Implementar acciones inclusivas en la enseñanza universitaria constituye uno de los mayores retos educativos en la actualidad. En el presente trabajo, y por medio de una revisión documental, analizamos las políticas de inclusión de las universidades públicas hondureñas. Entre los resultados encontramos el uso de un concepto de inclusión educativa destinado a estudiantes en situación de discapacidad. Discutimos, así mismo, los diferentes desafíos que se imponen en Honduras visto el incipiente desarrollo de los procesos inclusivos en la educación superior. Esto nos lleva a estudiar el concepto de inclusión y considerarlo mediante una perspectiva más amplia. Concluimos que hablar de inclusión educativa implica, a su vez, hablar de democracia y justicia social.

\section{PALABRAS CLAVE:}

democratización de la educación, educación superior, Honduras, justicia social

Today, a diverse student body attends the educational centers. As part of this diversity are those who belong to groups that are systematically violated. Implementing inclusive actions in higher education makes up one of the greatest educational challenges in the present day. In this work, and through a documentary review, we analyze the inclusion policies of Honduran public universities. Among the results we find the use of a concept of educational inclusion aimed at students with disabilities. We also discuss the different challenges that are imposed in Honduras, given the incipient development of inclusive processes in higher education. This leads us to study the concept of inclusion and consider it from a broader perspective. We conclude that talking about educational inclusion implies, in turn, talking about democracy and social justice.

\section{KEYWORDS:}

Democratization of Education, Higher Education, Honduras, Social Justice 



\section{Introducción}

La cobertura en cuanto a la educación superior aumentó de manera drástica durante el siglo XX (Rama, 2009). Con esto, la enseñanza universitaria dejó de ser exclusiva para la élite y se abrieron posibilidades de acceder a ella para una multiplicidad de estudiantes. Aunque no es la temática de este artículo, es determinante manifestar que esa masificación ha sido puesta en entredicho en el contexto de las políticas neoliberales impulsadas en América Latina (Cuño-Bonito, 2016; Guerrero; Soto-Arango, 2019; Espinoza; González, 2010). Para los intereses del presente artículo, destacamos que esta ampliación de matrícula trajo una mayor diversidad en las aulas universitarias, situación que dio pie a nuevos desafíos para la educación superior en el siglo XXI.

Las desigualdades en los centros de enseñanza superior sobreviven hasta hoy en lo que respecta a ingreso y promoción, pese a que se han creado políticas educativas dirigidas hacia todo el estudiantado (Bhopal, 2017). La perspectiva de América Latina se posa en países con más altos ingresos, en los que se han realizado intentos por disminuir las desigualdades en sus sistemas educativos (Hughes, 2015).

El sistema de educación universitaria en Honduras devela carencias tanto en cobertura como en equidad. La respuesta, se ha planteado desde una mirada al sistema educativo como un bien público al que debiera existir amplio acceso (Calderón, 2011). En las universidades hondureñas, cada día vemos la presencia de estudiantes que pertenecen a grupos vulnerados: alumnado en situación de discapacidad, afrodescendientes, pertenecientes a pueblos indígenas o colectivos de diversidad sexual forman parte de esa diversidad que necesitamos comprender, y para la cual corresponde generar prácticas educativas inclusivas encaminadas a disminuir las problemáticas relacionadas con tales grupos (Bhopal, 2017).

Una mirada más amplia de la inclusión implica dar un paso más allá del acceso; reorientar la admisión es solo el comienzo hacia una verdadera inclusión educativa (Mwaipopo; Lihamba; Njewele, 2011). Se deben ofrecer garantías para que los pertenecientes a los grupos nombrados puedan tener una mejor permanencia en su vida universitaria y, por ende, una promoción exitosa.

Identificar los factores que producen la exclusión en la enseñanza superior es determinante para implementar iniciativas que posibiliten disminuirla o erradicarla. Esto conduce a hacer el intento de generar una verdadera inclusión educativa (Castro; Rodríguez-Gómez; Gairín, 2016). Las universidades pueden contribuir con la formación de comunidades que respondan a las demandas inclusivas de toda la población universitaria y de la sociedad en general (Armstrong; Cairnduff, 2012). 
Con lo dicho, la finalidad de este artículo es analizar, desde una perspectiva teórica, las políticas de inclusión educativa de las universidades públicas hondureñas, tomando como referencia al ente rector de la enseñanza universitaria de Honduras.

\section{Un breve acercamiento teórico sobre la inclusión educativa}

El concepto de inclusión educativa en el ámbito de educación terciaria ha sido utilizado como equivalente al de equidad en el acceso (García; Adrogué, 2019; Espinoza; González, 2010). Quizás por esta razón, en muchos casos se tiende a generar cierta confusión en los conceptos (Llomovatte; Naidorf, 2014). Como un modo de resolver ese problema, en este texto comprendemos la inclusión educativa más allá de una justicia distributiva - que puede ser el concepto de equidad- (Calvo, 2013; Suárez, 2018).

La inclusión educativa tiene como finalidad promover el derecho a la participación y garantizar al estudiantado universitario una formación de calidad. Desde esta mirada, la diversidad toma un gran valor en los entornos inclusivos (Moriña, 2017). La diversidad constituye parte fundamental para la creación de espacios democráticos de aprendizaje, posibilitando la construcción de una sociedad más justa (Paz-Maldonado, 2020; Gaete; Luna, 2019; Díaz; Druker, 2007).

La literatura actual sobre inclusión educativa analiza particularmente este planteamiento a partir de una visión de procesos positivos, y escasamente desde los obstáculos que impiden su desarrollo. Es posible que esta sea la causa por la que podemos encontrar pocos planteamientos teóricos que cuestionan las ideas mayormente aceptadas acerca de dicha concepción (Kovač; Vaala, 2019).

Los significados y apreciaciones acerca de la inclusión educativa plantean tensiones y diferencias vinculadas con la democracia, las reformas educacionales y la justicia social. Sabemos que la mantención de las políticas de inclusión en las instituciones tiende a ser fuente de contradicciones, incluso en aquellas universidades que ya han optado por un camino en ese sentido (Leo; Barton, 2006). La comprensión de la justicia social y la democracia son aspectos de difícil interpretación. Por esa razón pensamos que es necesario atender la inclusión, la democracia y la justicia social como procesos en construcción (Paz-Maldonado, 2020; Gaete; Luna, 2019; Silva-Peña; Diniz-Pereira; Zeichner, 2017; Silva-Peña, 2021; Silva-Peña; Paz-Maldonado, 2019). Además, pueden ser espacios que sean conquistados día a día, tanto en el ámbito cotidiano como en la macroestructura.

Con el propósito de ser inclusivos, debemos comenzar por aprender a trabajar de forma cooperativa con los demás y promover estrategias que sean propias de cada 
centro educativo y de las comunidades, sin imponerlas desde otros contextos. Esto exige un compromiso y análisis crítico con nosotros mismos (Armstrong; Cairnduff, 2012). Para llevar a cabo prácticas inclusivas en los entornos de enseñanza deben comprenderse en términos conceptuales la inclusión educativa, las actitudes hacia dichas prácticas y el modo en que los centros educativos pueden preparar al profesorado para lograr incluir al estudiantado (Bemiller, 2019). La participación y la inclusión son formas distintas y complementarias que facilitan el aprendizaje (Quick; Feldman, 2011), y esta última no se limita a modificar los sistemas o las formas administrativas de implementarla, pues se relaciona con la dignidad y el bienestar de cada individuo (Reindal, 2015). La inclusión es la participación, la presencia y el logro de todo el estudiantado (Ainscow, 2007).

En este artículo se entiende por inclusión educativa aquellas acciones que posibiliten la democratización de la educación, permitan la participación de todo el estudiantado sin ningún tipo de discriminación, promuevan cambios profundos en la enseñanza universitaria y reconozcan a los diferentes grupos sociales que asisten con regularidad a las universidades públicas de Honduras (Paz-Maldonado, 202O; 2021). Respecto a las políticas de inclusión educativa, consideramos que son todos aquellos elementos legales y distintos mecanismos que facilitan generar una verdadera inclusión en los espacios educativos. De igual manera, propician la creación de una serie de apoyos en busca de atender las necesidades de cada estudiante.

\section{El contexto hondureño}

Honduras es un país centroamericano, con una población de casi 9 millones de habitantes y una superficie terrestre cercana al $10 \%$ del territorio colombiano, o casi el $5 \%$ del argentino. En el entorno hondureño se vivencia una alarmante crisis económica, política y social. En cuanto a pobreza, las cifras son desgarradoras: 68,8 $\%$ de la población se encuentra bajo esta línea y 44,2 \%, en extrema pobreza (Foro Social de la Deuda Externa y Desarrollo de Honduras [Fosdeh], 2018). La nación es calificada como una de las más desiguales de Centroamérica y el planeta (Alas; Moncada, 2010) superada únicamente por algunas naciones del continente africano (Justo, 2016).

En el sistema educativo hondureño se sufre una profunda crisis estructural, dado que en el país se han implementado una serie de medidas neoliberales con miras a lograr la privatización en este y otros sectores. Todo esto en un contexto en el que aumentan las denuncias sobre violaciones a los derechos humanos, hay un descenso en los niveles de seguridad y se genera un mayor cuestionamiento al 
gobierno (Paz-Maldonado; Díaz-Pérez, 2019). En ese escenario, miles de habitantes abandonan la nación bajo el sueño de un futuro mejor (Moreno, 2018).

Bajo el complejo panorama hondureño, analizamos la problemática que significa la inclusión educativa. Por ello somos conscientes del tremendo desafío que enfrentan las universidades hondureñas, en especial las de carácter público. Este tipo de instituciones no solo tienen un deber social transformativo porque son receptoras de aportes gubernamentales, sino que, además, atienden a la población vulnerada. Como ejemplo tenemos a la Universidad Nacional Autónoma de Honduras, que bajo mandato constitucional tiene la obligación de ser parte del proceso transformativo del país (Decreto n. ${ }^{\circ} 131,1982$ ).

\section{La educación superior pública en Honduras}

El año 2019 marcó un periodo de cambios en la educación superior de Honduras, puesto que el Consejo de Educación Superior (CES) aprobó el funcionamiento de un nuevo establecimiento privado. En la actualidad, el país cuenta con 21 instituciones de educación superior, 6 de ellas de carácter público y el resto privadas (Rodríguez, 2019). Asimismo, en el artículo n. ${ }^{\circ} 160$ de la Constitución Política de la República se establece que "la Universidad Nacional Autónoma de Honduras es una Institución autónoma del Estado, con personalidad jurídica, goza de la exclusividad de organizar, dirigir y desarrollar la educación superior y profesional" (Decreto n. ${ }^{\circ} 131,1982$ ). Los demás establecimientos de enseñanza universitaria (públicos y privados) que existen en el país deben regirse por los lineamientos establecidos en la máxima casa de estudios. En el cuadro 1 se listan los centros educativos estatales.

CUADRO 1 Universidades públicas de Honduras

Universidades

Fechas de fundación

\begin{tabular}{lc} 
Universidad Nacional Autónoma de Honduras (UNAH) & 1847 \\
\hline Universidad Pedagógica Nacional Francisco Morazán (UPNFM) & 1988 \\
\hline Universidad Nacional de Agricultura (UNAG) & 1994 \\
\hline Universidad Nacional de Ciencias Forestales (UNACIFOR) & 1994 \\
\hline Universidad de Defensa de Honduras (UDH) & 2005 \\
\hline Universidad Nacional de la Policía de Honduras (UNPH) & 2010
\end{tabular}


Si bien es cierto que existe una diversidad de instituciones de educación superior, las Universidades Nacional Autónoma de Honduras y la Pedagógica Nacional Francisco Morazán concentran el $60 \%$ de la matrícula $-44 \%$ y $15 \%$, respectivamente- (Dirección de Educación Superior, 2017). También es determinante notar que el Estado tiene como obligación destinar el $6 \%$ del presupuesto al sostenimiento, desarrollo y crecimiento de la Universidad Nacional Autónoma de Honduras (Decreto n. ${ }^{\circ} 131,1982$, art. 161). Este punto es esencial para comprender el peso de dicha institución en el sistema universitario hondureño. Asimismo, existen críticas permanentes en torno al financiamiento, puesto que el sistema universitario público se ve limitado económicamente para impulsar acciones que busquen contrarrestar las diversas problemáticas existentes en la sociedad (una de ellas radica en generar la inclusión educativa en la enseñanza universitaria).

\section{Método}

Para realizar este trabajo se llevó a cabo un escrutinio de todas las leyes, reglamentos y documentos que incorporan la temática de inclusión o la diversidad y que al momento de elaborar este artículo se encuentran vigentes. Realizamos un rastreo en las páginas oficiales de los organismos educativos del Estado y de la Universidad Nacional Autónoma de Honduras, puesto que es el ente rector de la enseñanza superior y, como lo expusimos, concentra la mayoría de la matrícula en el país. El objetivo central fue considerar los aspectos de inclusión que están presentes en la documentación revisada, de tal modo que se pudiera avanzar en el análisis posterior, concentrándonos de modo principal en los distintos desafíos para generar la inclusión educativa en las universidades públicas hondureñas.

Encontramos 14 documentos, que clasificamos en tres tipos:

1. Documentos de carácter nacional que implican a toda la educación del país. Bajo este concepto consideramos las siguientes normativas nacionales:

a. Constitución Política de la República de Honduras (Decreto No 131, 1982).

b. Ley de Equidad y Desarrollo Integral para las Personas con Discapacidad (Decreto n. $\left.{ }^{\circ} 160-2005,2005\right)$.

c. Ley Fundamental de Educación (Decreto n. ${ }^{\circ}$ 262-2011, 2012).

d. Reglamento General de la Ley Fundamental de Educación (Acuerdo Ejecutivo n. ${ }^{\circ}$ 13)8-SE-2014, 2014).

e. Reglamento de Educación Inclusiva para Personas con Discapacidad, Necesidades Educativas Especiales y Talentos Excepcionales (Acuerdo Ejecutivo n. ${ }^{\circ} 1365$-SE2014, 2014). 
f. Ley para el Desarrollo de la Educación Alternativa No Formal (Decreto n. ${ }^{\circ} 313^{-}$98, 1999).

g. Ley de Evaluación, Acreditación y Certificación de la Calidad y Equidad de la Educación (Decreto n. ${ }^{\circ}$ 265-2013, 2014).

h. Código de la Niñez y la Adolescencia (Decreto n. ${ }^{\circ} 73^{-96}, 1996$ ).

i. Ley Marco para el Desarrollo Integral de la Juventud (Decreto n. ${ }^{\circ} 260,2005$, 2006).

j. Ley de Igualdad de Oportunidades para la Mujer (Decreto n. ${ }^{\circ} 34,2000,2000$ ).

k. Ley de Educación Superior (Decreto n. ${ }^{\circ}$ 142-89, 1989).

2. Documentos de carácter sectorial: corresponden a la normativa general de la educación superior de Honduras. Como parte de esta clasificación hemos incorporado los siguientes:

a. Constitución Política de la República de Honduras (Decreto n. ${ }^{\circ} 131,1982$ ).

b. Ley de Educación Superior (Decreto n. ${ }^{\circ}$ 142-89, 1989).

c. Ley de Equidad y Desarrollo Integral para las Personas con Discapacidad (Decreto n. $\left.{ }^{\circ} 160-2005,2005\right)$.

3. Documentos de carácter local. En este caso se incluyen todas las normativas que rigen de modo específico a la Universidad Nacional Autónoma de Honduras. Para ello, hemos incluido los siguientes:

a. Ley Orgánica de la Universidad Nacional Autónoma de Honduras (Decreto n. ${ }^{\circ}$ 209-2004, 2005).

b. Modelo Educativo de la UNAH (2009).

c. Normas Académicas de la UNAH (2015).

\section{Resultados: el desafío universitario de la inclusión educativa en Honduras}

La revisión de los documentos nos entregó como resultado una descripción básica en torno a la inclusión. Vemos que el desarrollo de políticas de inclusión en Honduras tiene un carácter inicial. De algún modo, esto nos entrega una posibilidad: la de establecer los desafíos que presentan las políticas públicas. Por este motivo, los hallazgos encontrados, aunque sean mínimos, son discutidos a partir de la relación con la literatura. 
Organizamos el análisis de las distintas políticas, normativas y propuestas en torno a 5 ejes. Las dimensiones de este último se compusieron desde la perspectiva comentada en la introducción. Así, planteamos 5 ejes de discusión: implementar programas de inclusión; formación del profesorado; creación de políticas educativas inclusivas; promoción de la inclusión educativa; y democratizar la enseñanza superior pública. Además, agregamos un último tema, pilar en el desarrollo de las políticas mencionadas: el financiamiento. La temática de financiamiento necesita ser discutida a la luz de los diferentes desafíos que enfrenta la inclusión en la educación superior.

Debido al entorno en el que se desempeñan las universidades públicas de Honduras, son diversos los retos que estas deben enfrentar para lograr implementar con éxito la inclusión educativa en las distintas disciplinas y espacios de aprendizaje. Entre estos desafíos se pueden señalar los siguientes:

Implementación de programas de inclusión y atención a la diversidad. Los programas de este tipo han sido creados en especial para atender al estudiantado en situación de discapacidad a través del Programa de Servicios a Estudiantes con Necesidades Especiales (Prosene), que está bajo la dirección de la Universidad Nacional Autónoma de Honduras. En el caso de la Universidad Pedagógica Nacional Francisco Morazán se ha configurado el Proyecto de Atención a la Diversidad (Paz-Maldonado, 2018). Las instituciones restantes no han participado en este tipo de iniciativas, con lo que al menos $40 \%$ de la matrícula nacional no cuenta con atención alguna frente a estas situaciones. Por tanto, es necesario que la mayor parte de universidades públicas instaure programas de inclusión y atención a la diversidad.

Formación del profesorado en inclusión educativa. El profesorado a cargo de la docencia en las universidades públicas hondureñas, en general, ha recibido una formación incipiente en lo que se refiere a la atención a los colectivos que se encuentran en condiciones de vulnerabilidad, puesto que esta temática ha estado ausente en las mallas curriculares de los distintos programas de formación inicial y permanente. Así, no fue posible hallar propuestas sistemáticas de formación del profesorado en la atención al alumnado en situación de discapacidad, afrodescendiente e indígena, o a la diversidad sexual. Los programas de profesionalización docente son vitales en el fortalecimiento y desarrollo de competencias docentes con miras a generar inclusión educativa. Es necesario abordar dicho componente en la capacitación de los académicos que laboran en los centros de enseñanza superior públicos de Honduras.

Creación de políticas educativas inclusivas. A pesar de que la inclusión educativa en el mundo ha tenido cierto desarrollo, en Honduras aún no existen políticas univer- 
sitarias que faciliten el camino hacia ella. Por tanto, son pocas las acciones que se realizan en estos establecimientos. Consideramos necesarias las modificaciones en la relación con la comunidad estudiantil para revertir esta situación de desmedro. Así, un enfoque de inclusión basado en la participación daría lugar a democratización y compromisos institucionales. Sobre este punto, las políticas públicas y las características institucionales inciden en la vida académica del estudiantado (Castro et al., 2016); son esenciales en el funcionamiento efectivo de las universidades y una transformación en y desde la educación.

Promoción de la inclusión educativa en los espacios universitarios. Visto el desconocimiento actual de las concepciones de inclusión, sería determinante realizar campañas de sensibilización en el entorno universitario. Esto podría llevarse a cabo a través de mesas de diálogo donde los colectivos puedan dar a conocer sus peticiones para construir una universidad más inclusiva, así como talleres conferencias, jornadas de concientización y distintas actividades que involucren la participación de dichos grupos junto con la comunidad universitaria en busca de generar entornos tolerantes hacia la diversidad, dado que actualmente existe discriminación hacia diferentes grupos en condición de vulnerabilidad (Paz-Maldonado, 2018).

Democratizar la enseñanza superior pública. Las universidades públicas de Honduras tienen en su espíritu la participación de toda la comunidad universitaria en la toma de decisiones. Hoy existe una serie de tensiones debido al incumplimiento de este aspecto, lo que ha desencadenado acciones de protesta en los diferentes establecimientos estatales. Nuestra propuesta es impulsar una formación de profesionales que se base en los conceptos de la justicia social. Entendemos que el contexto hondureño amerita la formación de profesionales que reviertan las situaciones de injusticia e impunidad en las que se encuentra el país. El derecho a la educación es un comienzo fundamental, al igual que las miradas en torno a la equidad en el acceso, aunque, tal vez, sea necesario un mayor esfuerzo institucional.

Los sistemas de admisión universitarios tendrán que analizar nuevos mecanismos para potenciar el acceso del alumnado en condición de vulnerabilidad, debido a que las pruebas aplicadas son excluyentes. Un ejemplo de esto es el estudiantado en situación de discapacidad: demanda un ingreso especial, pero no paternalista ni segregador, relacionado con las condiciones con que cuenta cada universidad.

Asignación de recursos financieros para impulsar la inclusión educativa. Por ser un problema de reciente abordaje, las universidades públicas en la actualidad no reciben un presupuesto suficiente en el ámbito de la inclusión. El enfoque tradicional está 
basado en el acceso y no se vislumbran acciones afirmativas que permitan construir un espacio más justo para las personas pertenecientes a los colectivos vulnerados. Por tanto, es necesario concientizar a los tomadores de decisiones de tales centros, con la finalidad de que promuevan iniciativas inclusivas y destinen cierta cantidad de fondos a ello. Por ejemplo, los pueblos indígenas y afrodescendientes, aparte de ser reconocidos como tales, requieren ayudas (y de modo específico, aquellos que solo dominan su lengua materna y no el español). En el caso del alumnado con preferencias sexuales diversas, y de modo puntual, los estudiantes trans, es imprescindible ofrecerles condiciones que disminuyan la discriminación por su nueva identidad de género.

\section{Discusión}

El propósito fundamental de este trabajo fue realizar una revisión de las políticas de inclusión en la educación superior pública de Honduras, tomando como referencia el organismo rector de la enseñanza universitaria. A través de un análisis documental de las políticas generadas en el país, consideramos que el desarrollo de la inclusión educativa es incipiente. Los escasos resultados, si bien muestran carencias del sistema educativo hondureño, nos entregan la posibilidad de abrir la discusión. Por tal motivo, en esta sección exploramos estos desafíos considerando la literatura internacional.

En primer lugar, pensamos que se requiere una serie de cambios en las prácticas educativas e institucionales que permitan la construcción de universidades más inclusivas (Moriña, 2017). Por ejemplo, para mejorar las trayectorias universitarias del estudiantado que es parte de las políticas de inclusión es imprescindible efectuar ciertas adaptaciones curriculares. Estos ajustes podrían diseñarse desde los principios del diseño universal de aprendizaje (Melero-Aguilar; Moriña; Perera, 2019) u otros. Sin embargo, más allá de la metodología utilizada, creemos que se hace necesario pensar una política nacional de atención a la diversidad en la educación superior. Así, el abordaje de esta perspectiva se ampliaría a todas las instituciones.

Creemos que las prácticas educativas e institucionales necesitan ser soportadas por una política nacional. Quizás, a nivel constitucional, también se hace necesario pensar en el amplio acceso a las aulas universitarias. Hablamos de política nacional como aquel techo bajo el cual se desarrollan los diversos programas en cada una de las instituciones de educación superior. Esta dirección permitiría construir dispositivos que mejoren las posibilidades de ingreso, permanencia y promoción del estudiantado que ha sido excluido de forma sistemática. Repensar la política pública tiene como 
objetivo escapar del asistencialismo, promoviendo la autodeterminación de las personas; en este sentido, entender la inclusión educativa a través de una perspectiva amplia es indispensable para el desarrollo de una política pública.

La participación directa del profesorado es determinante para llevar a cabo la inclusión educativa (Calvo, 2013). Las experiencias que el estudiantado construye al interior de las aulas guardan mucha relación con los procesos de formación docente (Bhopal; Rhamie, 2014). Un nuevo tipo de formación en el profesorado se hace trascendental para la generación de prácticas pedagógicas inclusivas, al tiempo que facilitaría tener una mirada ecológica sobre las reacciones de las personas ante las distintas situaciones que ocurren en las aulas de clase (Contreras, 2002).

A pesar de que la inclusión educativa es una temática de interés y está tomando fuerza en las universidades de diversos países, no todos los miembros que forman parte de la comunidad universitaria están abiertos a ella (Joseph; Kearney; Wilson, 2019). Esta podría ser una de las mayores dificultades que deban enfrentar los centros de enseñanza superior al impulsar la inclusión.

Entendemos que la inclusión educativa es aquella que favorece la democratización de la educación: permite la participación del alumnado, posibilita la realización de cambios en las universidades y da lugar al reconocimiento de los diversos colectivos que asisten con regularidad a estos establecimientos formativos. Para un proceso de desarrollo en condiciones similares a las de sus pares es necesaria la eliminación de barreras a los sectores excluidos (Love; Baker; Devine, 2017). Así, es la institución la que va acogiendo la diversidad y deja de lado una visión en la que existe una normalidad por alcanzar.

El contexto hondureño en la actualidad es complejo, dado que existen diferentes problemas políticos, sociales y económicos que afectan el desarrollo de enfoques como la inclusión educativa y la justicia social, problemáticas que repercuten en las instituciones de enseñanza superior pública. Por tanto, llevar a cabo acciones inclusivas no es nada fácil. Solo una academia responsable, con nuevas visiones, comprometida con los cambios en la sociedad y que reconozca la existencia de un estudiantado heterogéneo puede promover este tipo de reajustes en la formación universitaria.

El profesorado es una figura determinante en la realización de transformaciones de los establecimientos del nivel superior, dado que a través de sus propias prácticas pedagógicas puede aportar elementos significativos en la generación de la inclusión educativa; sin embargo, requiere preparación. Asimismo, es vital desarrollar diferentes competencias y poseer capacidades que permitan producir cambios personales para comprender lo que realmente significa incluir al estudiantado universitario.

Honduras es un país con altas tasas de desigualdad, con problemas estructurales que devienen en injusticia social y en el que la educación se considera un gasto. 
Por tal razón, pensamos que promover la inclusión educativa implica repensar la enseñanza universitaria desde una visión de justicia social (Silva-Peña et al., 2021; Silva-Peña et al., 2017; Silva-Peña; Paz-Maldonado, 2019; Silva-Peña, 2021), pues los establecimientos públicos en esta lógica neoliberal que impera en América Latina son los más vulnerables ante las amenazas de la privatización.

\section{Conclusiones}

Hecha una mirada global, llegamos a visibilizar la necesidad de repensar las concepciones hegemónicas sobre el concepto de inclusión, cuyo repunte discursivo contrasta con su escaso accionar. Podemos mencionar que el término es inexistente en varias normativas vigentes en el contexto hondureño, y en otras solo se toma en cuenta el alumnado en situación de discapacidad. Al respecto, consideramos que entender la diversidad mediante una visión amplia propicia la inclusión educativa. Asimismo, vemos la necesidad de comprender la diversidad, la heterogeneidad como un valor, como una posibilidad de crecimiento para las instituciones y quienes forman parte de ellas. En este sentido, cabe señalar que pertenecemos a una sociedad diversa, en lugar de afirmar que debemos "incluir a los diversos". El trabajo mancomunado en torno a la comprensión de una sociedad diversa también hace posible un país diferente.

De algún modo, la construcción y aplicación de políticas inclusivas en las aulas de educación superior tiene el potencial de constituirlas en espacios democratizadores (Gaete; Luna, 2019). Pensar en esta relación entre democracia e inclusión permite tomar en cuenta lo que señalan Ainscow y Miles (2008) en términos de considerarla una lucha activa contra la exclusión. Creemos necesario ampliar la mirada: en cada momento en que se constituyan programas, políticas y normativas en torno a la inclusión, necesitaremos una propuesta que vaya más allá de una atención técnica. Por tal razón, aludimos a los conceptos de justicia social y democratización de la sociedad como elementos centrales al pensar la inclusión educativa.

\section{Referencias}

Acuerdo Ejecutivo n. ${ }^{\circ}$ 1358-SE-2014 del 2014 (17 de septiembre), por el cual se establece el Reglamento General de la Ley Fundamental de Educación. Diario Oficial La Gaceta.

Acuerdo Ejecutivo n. ${ }^{\circ}$ 1365-SE-2014 del 2014 (17 de septiembre), por el cual se establece el Reglamento Educación Inclusiva Para Personas con Discapacidad, Necesidades Educativas Especiales y Talentos Excepcionales. Diario Oficial La Gaceta. 
Ainscow, $\mathrm{Mel}$ (2007). From Special Education to Effective Schools for all: A review of progress so far. In L. Florian (Ed.), The SAGE Handbook of Special Education, pp. 146-159. London: Sage.

Ainscow, Mel; Miles, Susie (2008). Por una educación para todos que sea inclusiva: ¿Hacia dónde vamos ahora? Perspectivas, 38(1), 17-45. Recuperado de https://bit.ly/2nWHdeC

Alas, Mario; Moncada, Germán (2010). Problemas de equidad en el sistema educativo hondureño. Revista Iberoamericana de Evaluación Educativa, 3(3), 135-151.

Armstrong, Daniel; Cairnduff, Annette (2012). Inclusion in Higher Education: Issues in University- School Partnership. International Journal of Inclusive Education, 16(9), 917928. https://doi.org/10.1080/13603116.2011.636235

Bemiller, Michelle (2019). Inclusion for All? An Exploration of Teacher's Reflections on Inclusion in Two Elementary Schools. Journal of Applied Social Science, 13(1), 1-15. https:// doi.org/10.1177/1936724419826254

Bhopal, Kalwant (2017). Addressing Racial Inequalities in Higher Education: Equity, Inclusion and Social Justice. Ethnic and Racial Studies, 4O(13), 2293-2299. https://doi.org/10.1080/ 01419870.2017.1344267

Bhopal, Kalwant; Rhamie, Jasmine (2014). Initial Teacher Training: Understanding "Race," Diversity and Inclusion. Race Ethnicity and Education, 17(3), 304-325. https://doi.org/10.1 o8o/13613324.2013.832920

Calderón, Rutilia (2011). El crecimiento y desarrollo de la educación superior en Honduras, una perspectiva desde la UNAH. Innovación Educativa, 11(57), 81-89.

Calvo, Gloria (2013). La formación de docentes para la inclusión educativa. Páginas De Educación, 6(1), 19-35.

Castro, Diego; Rodríguez-Gómez, David; Gairín, Joaquín (2016). Exclusion Factors in Latin American Higher Education: A Preliminary Analyze From University Governing Board Perspective. Education and Urban Society, 49(2), 1-19. https://doi. org/10.1177/0013124516630599

Contreras, José (2002). Educar la mirada y el oído. Percibir la singularidad y también las posibilidades. Cuadernos de Pedagogía, 311, 61-65.

Cuño-Bonito, Justo (2016). La universidad Latinoamericana en la encrucijada: amenazas, desafíos y soluciones. Revista Historia de la Educación Latinoamericana, 18(26), 241-277. https://doi.org/10.19053/o1227238.4374

Decreto n. ${ }^{\circ} 131$ de 1982 (11 de enero), por el cual se establece la Constitución Política de la República de Honduras. Diario Oficial la Gaceta. 
Decreto n. ${ }^{\circ}$ 142-89 de 1989 (17 de octubre), por el cual se establece la Ley de Educación Superior. Diario Oficial la Gaceta.

Decreto . $^{\circ}$ 160-2005 de 2005 (30 de septiembre), por el cual se establece la Ley de Equidad y Desarrollo Integral para las Personas con Discapacidad. Diario Oficial la Gaceta.

Decreto n. ${ }^{\circ}$ 209-2004 de 2005 (12 de febrero), por el cual se establece la Ley Orgánica de la Universidad Nacional Autónoma de Honduras. Diario Oficial la Gaceta.

Decreto n. ${ }^{\circ}$ 260-2005 de 2006 (16 de enero), por el cual se establece la Ley Marco para el Desarrollo Integral de la Juventud. Diario Oficial la Gaceta.

Decreto n. ${ }^{\circ}$ 262-2011 de 2012 (22 de febrero), por el cual se establece la Ley Fundamental de Educación. Diario Oficial la Gaceta.

Decreto n. ${ }^{\circ}$ 265-2013 de 2014 (17 de febrero), por el cual se establece la Ley de Evaluación, Acreditación y Certificación de la Calidad y Equidad de la Educación. Diario Oficial La Gaceta.

Decreto n. ${ }^{\circ} 313-98$ de 1999 (15 de febrero), por el cual se establece la Ley para el Desarrollo de la Educación Alternativa No Formal. Diario Oficial la Gaceta.

Decreto n. ${ }^{\circ} 34-2000$ de 2000 (28 de abril), por el cual se establece la Ley de Igualdad de Oportunidades para la Mujer. Diario Oficial la Gaceta.

Decreto n. ${ }^{\circ}$ 73-96 de 1996 ( 5 de septiembre), por el cual se establece el Código de la Niñez y la Adolescencia. Diario Oficial la Gaceta.

Díaz, Tatiana; Druker, Sofía (2007). La democratización del espacio escolar: una construcción en y para la diversidad. Estudios Pedagógicos, 33(1), 63-77.http://dx.doi.org/10.4067/So71807052007000100004

Dirección de Educación Superior (2017). Anuario Estadistico, UNAH. Recuperado de https:// des.unah.edu.hn/servicio-al-usuario/estadisticas/\#EducacionSuperior\%2O\#Estadisticas

Espinoza, Óscar; González, Luis (2010). Políticas y estrategias de equidad e inclusión en educación superior en América Latina: experiencias y resultados. ISEES: inclusión social y equidad en la educación superior, 7, 21-35. Recuperado de https:/dialnet.unirioja.es/ descarga/articulo/3777548.pdf

Foro Social de Deuda Externa y Desarrollo de Honduras - Fosdeh (2018). Infografia Hondurasy su realidad expresada en imágenes. Recuperado de https://es.scribd.com/document/472894293/ Infografia-Honduras-y-su-realidad-expresada-en-imagenes

Gaete, Alfredo; Luna, Laura (2019). Educación inclusiva y democracia. Revista Fuentes, 21(2), 161-175. 
García, Ana; Adrogué, Cecilia (2019). Equidad en el acceso y la graduación en la educación superior: reflexiones desde el Cono Sur. Archivos Analíticos de Políticas Educativas, 27(96), 1-38. https://doi.org/10.14507/epaa.27.3943

Guerrero, Sara; Soto-Arango, Diana (2019). La política educativa en torno a la masificación de la educación superior y su relación con el abandono universitario en Colombia. Revista Historia de la Educación Latinoamericana, 21(32), 109-136. https://doi.org/10.19053/01227238.9201

Hughes, Katie (2015). The social inclusion meme in higher education: are universities doing enough? International Journal of Inclusive Education, 19(3), 303-313. https://doi.org/10.10 8o/13603116.2014.930518

Joseph, Brianna; Kearney, Kelly; Wilson, Cynthia (2019). The Role of Educational Leaders: A Case for Inclusion in Institutions of Higher Education. Journal of Cases in Educational Leadership, 22(3), 3-15. https://doi.org/10.1177/1555458919847245

Justo, Marcelo (9 de marzo del 2016). ¿Cuáles son los 6 países más desiguales de América Latina? BBC Mundo. Recuperado de https://www.bbc.com/mundo/noticias/2016/o3/160308_ america_latina_economia_desigualdad_ab

Kovač, Velibor; Vaala, Birgit (2019). Educational Inclusion and Belonging: A Conceptual Analysis and Implications for Practice. International Journal of Inclusive Education, 1-15. https://doi.org/10.1080/13603116.2019.1603330

Leo, Elizabeth; Barton, Len (2006). Inclusion, Diversity and Leadership: Perspectives, Possibilities and Contradictions. Educational Management Administration \& Leadership, 34(2), 167-18o. https://doi.org/10.1177/1741143206062489

Llomovatte, Silvia; Naidorf, Judith (2014). Equity, Social Cohesion and Relevance in Higher Education. En European and Latin American Higher Education Between Mirrors (pp. 197-211), editado por António Teodoro y Manuela Guilherme. Rotterdam: Sense Publishers. https:// doi.org/10.1007/978-94-6209-545-8_14

Love, Matthew; Baker, Joshua; Devine, Stephanie (2017). Universal Design for Learning: Supporting College Inclusion for Students With Intellectual Disabilities. Career Development and Transition for Exceptional Individuals, 42(2), 1-6. https://doi. org/10.1177/2165143417722518

Melero-Aguilar, Noelia; Moriña, Anabel; Perera, Víctor-Hugo (2019). Acciones del profesorado para una práctica inclusiva en la universidad. Revista Brasileira de Educação, 24, 1-19. https:// dx.doi.org/10.1590/s1413-24782019240016

Moreno, Ismael (29 de octubre de 2018). La caravana: ¿Quiénes la empujan, qué factores internos la provocan, cómo situarnos? Radio Progreso. Recuperado de http://wp.radioprogresohn. net/la-caravana-quienes-la-empujan-que-factores-internos-la-provocan-como-situarnos/ ?fbclid=IwARoQfTxoBwcfuaKAy4bOBboKCow25t8NQRIto6C5zNCKg3jnfcySQXgQjQM 
Moriña, Anabel (2017). Inclusive Education in Higher Education: Challenges and Opportunities. European Journal of Special Needs Education, 32(1), 3-17. https://doi.org/10.1080/o885625 7.2016 .1254964

Mwaipopo, Rosemarie; Lihamba, Amandina; Njewele, Delphine (2011). Equity and Equality in Access to Higher Education: The Experiences of Students with Disabilities in Tanzania. Research in Comparative and International Education, 6(4), 415-429. https://doi.org/10.2304/ rcie.2011.6.4.415

Paz-Maldonado, Eddy (2018). Situación actual de la atención a la diversidad en la educación superior de Honduras. Revista Actualidades Investigativas en Educación, 18(3), 1-32.

Paz-Maldonado, Eddy (2020). Una aproximación teórica sobre la educación inclusiva en Honduras: avances, obstáculos y desafíos. Revista Brasileira de Educação Especial. 26(3), 371-386. https://doi.org/10.1590/1980-54702020v26eoo49

Paz-Maldonado, Eddy (2021). La inclusión educativa del estudiantado universitario en situación de discapacidad en Honduras. Ensaio: Avaliação e Políticas Públicas em Educação, 29(112), 738-76o. https://doi.org/10.1590/So104-403620210002902767

Paz-Maldonado, Eddy; Díaz-Pérez, Wilmer (2019). Educación para la paz: una mirada desde la Universidad Nacional Autónoma de Honduras. Innovación Educativa, 19(79), 171-195.

Quick, Kathryn; Feldman, Martha (2011). Distinguishing Participation and Inclusion. Journal of PlanningEducation and Research,31(3), 272-29o.https://doi.org/10.1177/0739456X11410979

Rama, Claudio (2009). La tendencia a la masificación de la cobertura de la educación superior en América Latina. Revista Iberoamericana de Educación, 50, 173-195. https://doi. org/10.35362/rie500668

Reindal, Solveig (2015). Discussing Inclusive Education: An Inquiry into Different Interpretations and a Search for Ethical Aspects of Inclusion Using the Capabilities Approach. European Journal of Special Needs Education, 31(1), 1-12. https://doi.org/10.108 o/o8856257.2015.1087123

Rodríguez, Elin (2019). Después de 14 años, Honduras cuenta con una nueva universidad. Presencia Universitaria. Recuperado de https://presencia.unah.edu.hn/noticias/despuesde-14-anos-honduras-cuenta-con-una-nueva-universidad/

Silva-Peña, Ilich (2021). Justicia social como eje de la Formación Inicial Docente post 18 de octubre. En Estallido Social en Chile. Lecturas sobre discriminación y desigualdad educativa (pp.207-221), editado por Ilich Silva-Peña; María Angélica Oliva; Óscar Espinoza; Eduardo Santa-Cruz. Santiago de Chile: Editorial UTEM.

Silva-Peña, Ilich; Hizmeri-Fernández, Julio; Hormazábal-Fajardo, Roxana; González-García, Gustavo; Rojas-Rodríguez, Bessie; Jara-Illanes, Enriqueta (2021). Repolitización de la 
formación inicial docente en un Chile post pandemia. En La Educación en Tiempos de Confinamiento. Perspectivas de Lo Pedagógico (pp. 511-536), editado por Marcela Romero Jeldres; Solange Tenorio Eitel. Santiago de Chile: Fondo Editorial UMCE. Recuperado de https://zenodo.org/record/4948987\#.YNXFUOhKhPY

Silva-Peña, Ilich; Paz-Maldonado, Eddy (2019). Una reflexión acerca de la indagación narrativa autobiográfica en formadores/as de docentes para la justicia social.Perspectiva Educacional, $58(2), 169-189$.

Silva-Peña, Ilich; Diniz-Pereira, Julio;Zeichner, Ken (2017). Justicia social. La dimensión olvidada de la formación docente. Santiago de Chile: Mutante Editores.

Suárez, Cecilia (2018). Permanencia de estudiantes indígenas en instituciones de educación superior en América Latina. (Tesis de doctorado). Universitat Autònoma de Barcelona, Barcelona, España. Recuperado de https://ddd.uab.cat/record/189683

Universidad Nacional Autónoma de Honduras - UNAH (2009). Modelo Educativo de la Reforma Universitaria. Honduras: UNAH.

Universidad Nacional Autónoma de Honduras - UNAH (2015). Normas académicas de la Universidad Nacional Autónoma de Honduras. Honduras: UNAH. 